\title{
EXPERIMENTOS DE ENSEÑANZA: UNA ALTERNATIVA METODOLÓGICA PARA INVESTIGAR EN EL CONTEXTO DE LA FORMACIÓN INICIAL DE DOCENTES \\ TEACHING EXPERIMENTS: A METHODOLOGICAL ALTERNATIVE TO RESEARCH IN \\ THE CONTEXT OF THE INITIAL TEACHER TRAINING
}

\section{Volumen 14, Número 3 \\ Setiembre - Diciembre}

pp. $1-20$

Este número se publicó el 30 de setiembre de 2014

Gabriela Valverde Soto

Revista indizada en $\underline{\text { REDALYC, }}$ SCIELO

Revista distribuida en las bases de datos:

CATÁLOGO DE LATINDEX, IRESIE, CLASE, DIALNET, DOAJ, E-REVIST@S, SHERPA/ROMEO, QUALIS, MIAR

Revista registrada en los directorios:

ULRICH'S, REDIE, RINACE, OEI, MAESTROTECA, PREAL, CLACSO 


\title{
EXPERIMENTOS DE ENSEÑANZA: UNA ALTERNATIVA METODOLÓGICA PARA INVESTIGAR EN EL CONTEXTO DE LA FORMACIÓN INICIAL DE DOCENTES \\ TEACHING EXPERIMENTS: A METHODOLOGICAL ALTERNATIVE TO RESEARCH IN THE CONTEXT OF THE INITIAL TEACHER TRAINING
}

\section{Gabriela Valverde Soto ${ }^{1}$}

\begin{abstract}
Resumen: En este artículo se presenta una descripción general de uno de los estudios frecuentemente realizados dentro del enfoque de la investigación de diseño, como son los experimentos de enseñanza; en particular, se centra en aquellos denominados Teacher Development Experiment (TDE). Se describen los principios que los fundamentan y sus características centrales, con base en una revisión de documentos sobre el tema. Este análisis de documentos sirvió de sustento para estructurar la metodología de un estudio desarrollado por la autora en la Universidad de Granada, durante el periodo comprendido entre 2008 y 2012. Como parte de las conclusiones de tal estudio, se argumenta el potencial de esta metodología de investigación para explorar el desarrollo del conocimiento matemático del futuro docente de educación primaria. Con este aporte se pretende promover el uso de esta opción metodológica en la investigación educativa, especialmente la desarrollada en el contexto de la formación inicial de docentes.
\end{abstract}

Palabras clave: DIDÁCTICA DE LA MATEMÁTICA, EXPERIMENTOS DE ENSEÑANZA, FORMACIÓN INICIAL DE MAESTROS, INVESTIGACIÓN DE DISEÑO, SUPERIOR, ESPAÑA

Abstract: This report presents a general description of one of the studies frequently done inside the approach of Design Research, the Teaching Experiments. It focuses in particular on the experiments called Teacher Development Experiment (TDE) and describes the principles that sustain them and their main characteristics. This article was done by analyzing papers on the subject. The document analysis was used to structure the methodology of a study conducted by the author at the University of Granada during the period between 2008 and 2012. As part of the conclusions, the author argued the potential of this research methodology to explore the development of the mathematical knowledge of future teachers of elementary education. The aim of this paper is to promote the knowledge and the use of this methodological alternative in the investigation in Education, particularly that one developed in the context of the initial teacher training.

Key words: DESIGN RESEARCH, DIDACTICS OF MATHEMATICS, INITIAL TEACHER TRAINING, TEACHING EXPERIMENTS, HIGHER EDUCATION, SPAIN

\footnotetext{
${ }^{1}$ Profesora e investigadora de la Escuela de Formación Docente de la Universidad de Costa Rica. Doctora en Didáctica de la Matemática por la Universidad de Granada, España. Licenciada en Enseñanza de la Matemática de la Universidad de Costa Rica.
}

Dirección electrónica: GABRIELA.VALVERDE@ucr.ac.cr

Artículo recibido: 27 de enero, 2014

Devuelto para corrección: 20 de junio, 2014

Aprobado: 31 de julio, 2014 


\section{Introducción}

El estudio del aprendizaje en entornos cotidianos en lugar de ambientes con condiciones artificiales, ha sido una necesidad imperante en la investigación educativa desde sus orígenes hasta la actualidad; como una respuesta a este reto surge la investigación de diseño (Collins, Josep y Bielaczyc, 2004). Diversos autores se refieren a este tipo de investigación mediante términos tales como: investigación (estudio) de diseño, investigación (estudio) basada en diseño, experimento ${ }^{2}$ de diseño o basado en diseño.

Este tipo de investigación ha venido refinándose con el tiempo y cabe destacar que en los últimos 15 años se ha escrito ampliamente al respecto, sus principios, características, objetivos y las prácticas que supone (Brown, 1992; Cobb, Confrey, diSessa, Lehrer y Schauble, 2003; Collins, Josep y Bielaczyc, 2004; Design-Based Research Collective, 2003; Molina, 2007; Molina, Castro, Molina y Castro, 2011). Sin embargo, en el contexto nacional poco se ha divulgado sobre el tema, de modo que con este trabajo se pretende aportar una caracterización de la investigación de diseño a través de la descripción de sus principios generales y de la descripción de un tipo de estudio frecuentemente realizado en este enfoque (Teacher Development Experiments o experimentos TDE). En el cierre del trabajo se aportan reflexiones y recomendaciones, derivadas de la experiencia de la autora, referentes al uso de la metodología TDE.

\section{La investigación de diseño}

De acuerdo con Molina et al. (2011) la investigación de diseño es un enfoque o paradigma de investigación, de naturaleza cualitativa desarrollado en el campo de las "Ciencias del aprendizaje". La investigación de diseño "persigue comprender y mejorar la realidad educativa a través de la consideración de contextos naturales en toda su complejidad, y del desarrollo y análisis paralelo de un diseño instruccional específico" (p.75). Según estos investigadores, su objetivo es: "Analizar el aprendizaje en contexto mediante el diseño y estudio sistemático de formas particulares de aprendizaje, estrategias y herramientas de enseñanza, de una forma sensible a la naturaleza sistémica del aprendizaje, la enseñanza y la evaluación" (p. 76).

\footnotetext{
${ }^{2}$ Desde el enfoque de la investigación de diseño, el uso del término experimento no guarda relación con su empleo en los estudios experimentales o cuasi-experimentales en los cuales se trabaja con grupos control y experimental.
} 
Tales razones permiten considerar la investigación de diseño como un enfoque metodológico eficaz en la investigación del aprendizaje y la enseñanza. Para Molina (2007), los estudios de diseño están dirigidos principalmente a comprender los procesos de enseñanza y aprendizaje en los que el propio investigador se encuentra implicado.

Aunque cada autor sugiere algunos matices distintos, en este artículo se adopta la postura propuesta por Confrey (2006, p.135), quien los define como "amplias investigaciones de interacciones educativas, contemplan el uso de un conjunto de tareas curriculares, cuidadosamente secuenciadas que estudian cómo algún campo conceptual o conjunto de competencias e intereses son aprendidas mediante la interacción de los alumnos, bajo una guía". Este investigador sostiene que la investigación de diseño pretende documentar los recursos y conocimientos que manifiestan los alumnos en la resolución de las tareas, las interacciones entre los estudiantes y profesores, la evolución de las concepciones y en general, cómo se realiza la enseñanza a lo largo de la experimentación.

Tabak (2004) sostiene que la investigación de diseño es apta para el estudio de la enseñanza y el aprendizaje en contextos naturales de formación; esta investigadora sustenta su postura basándose en una extensa revisión de bibliografía realizada por Turner y Meyer, quienes identificaron un conjunto de componentes esenciales necesarios para estudiar contextos naturales de aula. Según Tabak (2004), la investigación basada en diseño satisface tales componentes y lo argumenta indicando que:

- El estudio del contexto de clase requiere de la investigación de más de una variable a la vez. Los investigadores implicados en la investigación de diseño comparten la idea de que el aprendizaje es un fenómeno que se desarrolla en medio de la coacción de múltiples factores e interacciones. Uno de los propósitos de la investigación de diseño es estudiar cómo distintas condiciones de la clase apoyan el aprendizaje.

- El contexto del aula requiere que el programa de investigación incorpore un componente cualitativo. El conocimiento producido a través del componente empírico de los métodos de la investigación de diseño, incluyen normalmente una descripción de cómo se desarrolla el aprendizaje día a día a través de las interacciones del aula. Este proceso implica métodos cualitativos de recolección de datos que permitan elaborar una descripción de la trayectoria que se ha seguido para conducir el aprendizaje de los individuos y del grupo. 
- Un estudio sobre el contexto cotidiano del aula debería intentar responder a las cuestiones ¿cómo sucede? y ¿por qué sucede algo? además del ¿qué sucede? La investigación de diseño acopia información acerca del aprendizaje y los medios a través de los cuales este se generó.

- El estudio del contexto del aula requiere que el investigador se encuentre presente en el aula. Uno de los distintivos de la investigación basada en diseño es la colaboración entre los participantes del entorno educativo dentro del cual se desarrolla el estudio.

Este tipo de investigación está siendo ampliamente aplicado en la investigación educativa, particularmente en el campo de la didáctica de la matemática (Molina, 2007); esta investigadora sustenta tal afirmación indicando la difusión de los estudios de diseño en diversas publicaciones y colectivos de investigadores, como el grupo Design-Based Research Collective.

\section{Características de la investigación de diseño}

Los experimentos de diseño fueron desarrollados como una manera de realizar investigación para evaluar y refinar diseños educativos basados en principios teóricos derivados de investigaciones previas (Collins et al., 2004). Según Molina et al. (2011) estos estudios se pueden caracterizar como se indica a continuación:

- La investigación de diseño se realiza en contextos naturales de enseñanza y aprendizaje, la complejidad de estos entornos se traduce en la implicación de múltiples variables, muchas de las cuales no se pueden controlar. Por ejemplo: cantidad y diversidad del estudiantado, características del centro educativo, prácticas educativas tradicionales que han prevalecido en una institución, tiempo facilitado a los investigadores para desarrollar el estudio, entre otras. En este sentido, los estudios de diseño se realizan en condiciones naturales, por ejemplo si se hace en un grupo de escolares no se realiza una selección de ellos, sino que se consideran a todos los estudiantes y las sesiones de aplicación se desarrollan en el mismo lugar, con el mismo horario y bajo las condiciones normales de ese grupo.

- Combina 2 propósitos: el diseño de situaciones o ambientes de aprendizaje y enseñanza, y el desarrollo de teorías, o como afirma el Colectivo de Investigación Basada en Diseño (Design-Based Research Collective, 2003), "proto-teorías". Este adjetivo se refiere a que las teorías desarrolladas son específicas a un dominio de 
aprendizaje y son explicativas de la actividad del diseño. Como señalan Molina et al. (2011), estas teorías basadas en hechos empíricos son esenciales para la mejora de la educación, debido a que son útiles para detectar regularidades o patrones en los complejos contextos en los que tienen lugar.

- La investigación y el desarrollo configuran un ciclo continuo de diseño de intervención (planificación), puesta en práctica, análisis y rediseño. Según Kelly (citado en Molina et al., 2011), en la toma de decisiones sobre la planificación, el aprendizaje de los alumnos no es el único factor determinante, ya que se pueden considerar otros factores de carácter práctico, participativos, políticos, entre otros. Cuando algunos aspectos del diseño no funcionan como se ha planificado, el equipo investigador debe tomar en cuenta diferentes opciones para mejorar la puesta en práctica de lo planificado y decidir sobre cambios en este, tantas veces como sea necesario. Una consecuencia del carácter cíclico se refiere a que tales estudios implican 2 niveles de análisis: uno preliminar, de los datos que se recogen sesión tras sesión, y el final, de toda la información recabada, y que se realiza al terminar todo el proceso de implementación.

- La investigación debe dar cuenta (explicar) de cómo y por qué funcionan los diseños educativos en contextos reales. No debe limitarse a documentar su éxito o fracaso, propio de la evaluación del producto. La investigación de diseño, afirman Cobb, Confrey, DiSessa, Lehrer, y Schauble (2003), pretende explicar por qué funcionan los diseños y sugerir cómo pueden adaptarse a nuevas circunstancias.

- El desarrollo de la investigación debe apoyarse en métodos que permitan constatar (y dar cuenta de) las conexiones de los procesos de puesta en operación con resultados de interés.

- Una de las limitaciones fundamentales en la investigación de diseño se refiere a la gran cantidad de datos que se recogen, y la mayoría procede de grabaciones de audio y (o) video de las intervenciones y del trabajo de los estudiantes, las cuales se acopian para comprender con detalle qué es lo que sucede. Este factor incide en la dificultad de llevar a cabo las investigaciones de diseño.

Los participantes, el espacio por observar, la duración del experimento, entre otras condiciones, conducen a establecer variaciones entre los estudios que se enmarcan en la 
investigación de diseño. Cobb, Confrey, DiSessa, Lehrer y Schauble (2003), indican que existen distintos tipos de experimentos de diseño, entre los cuales destacan:

- Experimentos de diseño "uno a uno", en los que un equipo de investigación conduce una serie de sesiones de enseñanza con un pequeño número de estudiantes; el objetivo es crear a pequeña escala la ecología del aprendizaje en el aula ordinaria, de modo que pueda ser estudiada con mayor profundidad y detalle (Cobb y Steffe, 1983; Steffe y Thompson, 2000).

- Experimentos con el grupo, en el cual un equipo de investigación colabora con un profesor (que puede ser uno de los miembros) y el equipo asume la responsabilidad de la enseñanza (Cobb, 2000; Confrey y Lachance, 2000; Gravemeijer, 1994).

- Experimentos sobre el desarrollo del conocimiento de profesores en activo, en los cuales los investigadores colaboran con los profesores para apoyar el desarrollo de una comunidad profesional.

- Experimentos sobre el desarrollo del conocimiento de profesores en formación, en los cuales un equipo de investigación ayuda, organiza y estudia la formación de los futuros docentes (Simon, 2000).

En síntesis, este enfoque de investigación va más allá del mero diseño y prueba de intervenciones particulares. Las sesiones de implementación incluyen determinados supuestos y exigencias específicas teóricas sobre la enseñanza y el aprendizaje, reflejan un compromiso para comprender las relaciones entre teoría, plan de acción diseñado y práctica, al mismo tiempo que el análisis previsto de cada sesión específica puede contribuir a elaborar teorías localizadas sobre la enseñanza y aprendizaje de un contenido específico. Este aspecto lo distingue de otros enfoques o metodologías como la investigación-acción.

\section{Criterios de evaluación de los estudios de diseño}

La validez y la fiabilidad, entre otras, son condiciones necesarias de toda investigación; sin embargo, en los experimentos de diseño estas cualidades se tratan de forma notoriamente diferente a como se hace en la investigación experimental, en la que "el investigador deliberadamente controla y manipula las condiciones que determinan los hechos en lo que está interesado" (Cohen y Manion, 2002, p. 243). Molina et al. (2011) señalan que autores tales como Cobb, Gravemeijer o Confrey, han abordado cuestiones 
relacionadas con la evaluación de la calidad de los estudios de diseño, entre las cuales están la fiabilidad, replicabilidad, capacidad de generalización y utilidad.

En los estudios de diseño la fiabilidad se evalúa con base en la medida en la que el análisis ha sido sistemático, los criterios asumidos en el análisis son explícitos, las argumentaciones o conclusiones finales proceden de razones que se han ido construyendo a lo largo del estudio, y si el análisis ha sido criticado por otros investigadores (Molina et al., 2011). Las condiciones indicadas fundamentan la generación de interpretaciones y conclusiones consistentes en relación con los datos recogidos sobre el objeto de estudio en la experimentación.

La replicabilidad se refiere a la posibilidad de repetir una investigación original: si se produce un resultado positivo, es decir, similar al encontrado inicialmente, se incrementa la confianza depositada en la investigación original; por el contrario, si se produce un resultado negativo, eso sugiere, o bien que los resultados iniciales se produjeron por azar (efecto del azar), o bien que ha habido una falta de control de variables que han contaminado el trabajo original (efecto del control).

De acuerdo con Molina et al. (2011), en la investigación de diseño la replicabilidad consiste en: Los aspectos del proceso de aprendizaje estudiado que pueden repetirse potencialmente en otros contextos o situaciones. Realizando estudios posteriores que utilicen el modelo obtenido como material conceptual a ser reorganizado, el modelo elaborado será sustituido por otro más avanzado (p. 79).

Este proceso contribuye a la comprobación de los resultados del experimento de diseño realizado inicialmente y al desarrollo del modelo teórico, el cual podría aplicarse en otras circunstancias, incidiendo en un aumento de la validez externa del estudio, es decir, en su capacidad de generalización, la cual se refiere a la extensión y forma en que los resultados de un experimento pueden generalizarse a diferentes sujetos, poblaciones, lugares, experimentadores. Sin embargo, en la investigación de diseño: "La capacidad de generalización no está condicionada por la representatividad de la muestra, sino íntimamente relacionada con la replicabilidad e implica que otros serán capaces de usar los productos que deriven de él para promover aprendizaje en otros contextos" (Molina et al., 2011, p. 79).

Otro criterio relacionado con la evaluación de este tipo de estudios, que ha sido considerado por los investigadores consultados, se refiere a la utilidad. En consecuencia, es fundamental explicitar las aportaciones e implicaciones de la investigación en la 
enseñanza. Este aspecto incide indudablemente en la capacidad de estos estudios para acercar la teoría y la práctica.

\section{Experimentos del desarrollo del conocimiento del profesor ${ }^{3}$ (Teacher Development Experiment TDE)}

La metodología TDE es una adaptación y extensión de los experimentos de enseñanza y de los experimentos con "todo el grupo" (whole-class experiments). Sin embargo, los estudios TDE, a diferencia de las otras 2, consideran la posibilidad de abordar distintos tipos de conocimientos, no solo el de las matemáticas.

Según Simon (2000), el término Teacher Development Experiment (TDE) es un intento de distinguirlo de los experimentos de enseñanza, aunque reconoce que estos últimos son un elemento central de los estudios TDE. Estudian el desarrollo del conocimiento del profesor en formación o en servicio, y se fundamentan en los principios de los experimentos de enseñanza (Cobb y Steffe, 1983; Steffe y Thompson, 2000), lo que significa que un equipo de investigadores estudia el desarrollo del conocimiento a la vez que lo promueve como parte de un ciclo continuo de análisis e intervención. Las investigaciones TDE también contemplan el estudio de casos.

El profesor-investigador promueve el desarrollo del conocimiento a través de actividades preparadas para el grupo. En todas las sesiones es preciso recoger la información mediante grabaciones de audio y (o) video. Después de las intervenciones el profesor-investigador se encuentra con otros investigadores para analizar la sesión previa, generar y modificar modelos del desarrollo del conocimiento del profesor y planear las siguientes intervenciones de enseñanza.

Mientras que, de modo general, los experimentos de enseñanza se han centrado en el desarrollo del conocimiento matemático, la metodología TDE se ha usado para generar también modelos del desarrollo del conocimiento didáctico, pues se reconoce que ambos tipos de conocimientos están interrelacionados. Así que los experimentos de enseñanza TDE utilizan la estructura de sus predecesores (teaching experiments $y$ whole-class experiments), pero amplían y modifican las áreas de concentración. Incluso en los cursos

\footnotetext{
${ }^{3}$ Desarrollo del Profesor (Teacher Development) se refiere a los cambios en los conocimientos, creencias, disposiciones y habilidades de los profesores en activo o en formación, que sustentan su capacidad para implementar exitosamente los principios de la educación matemática (Simon, 2000). En el estudio realizado por la autora de este artículo se ha adaptado el término a desarrollo del conocimiento del profesor.
} 
de matemáticas para profesores, la concentración es más amplia que en los experimentos de enseñanza con "todo el grupo" realizados por Cobb.

En síntesis, la metodología TDE es una adaptación y extensión de 2 tipos de estudios: los experimentos de enseñanza (teaching experiments) y los experimentos de enseñanza de "todo el grupo" (whole-class teaching experiments). A continuación se detallan las contribuciones de cada uno a la metodología TDE.

\section{Contribuciones de los experimentos de enseñanza a los experimentos TDE}

La metodología de los experimentos de enseñanza se ha originado con la intención de comprender el desarrollo de los conceptos en los niños, en áreas particulares de la matemática (Simon, 2000). De forma general, un experimento de enseñanza consiste en una secuencia de episodios de enseñanza en los que los participantes son normalmente un investigador-docente, uno o más alumnos y uno o más investigadores-observadores (Steffe y Thompson, 2000, citados en Molina et al., 2011). Seguidamente se presentan las características centrales de los experimentos de enseñanza, las cuales también están implicadas en los experimentos sobre el desarrollo del conocimiento del profesor.

Profesor-investigador: los experimentos de enseñanza han resultado ser una opción a los paradigmas en los cuales los investigadores son observadores o cuantificadores de situaciones "naturales" o "experimentales". El doble papel de investigador y profesor proporciona una oportunidad para que este desarrolle conocimiento a través de múltiples iteraciones de un ciclo de reflexión-interacción. Esta ruptura de la diferenciación entre docente e investigador está motivada por el propósito de experimentar de primera mano el aprendizaje y razonamiento de los alumnos (Kelly y Lesh, 2000; Steffe y Thompson, 2000).

Como parte del ciclo de reflexión-interacción, el investigador aplica a las interacciones con los estudiantes su conocimiento personal y aquel compartido por la comunidad de investigación, incluyendo conjeturas actuales sobre el fenómeno estudiado. La intuición y patrones de acción del investigador, los cuales no son parte de su conocimiento explícito, también contribuyen a la interacción. La interpretación de las interacciones por parte del investigador, le permite respaldar o constatar algunos aspectos de su conocimiento y desafiar otros, dando como resultado la modificación de lo que 
sabe. De modo que este ciclo es esencial en los experimentos de enseñanza, pues posibilita a los investigadores mejorar su conocimiento acerca de cómo aprenden los estudiantes. En general, se espera que todos los participantes del experimento construyan aprendizajes (Molina et al., 2011).

Análisis continuos y retrospectivos: los experimentos de enseñanza implican dos niveles de análisis de datos: (a) los continuos, que ocurren durante y entre las sesiones con los estudiantes, y (b) el retrospectivo, que se enfoca en el conjunto total de las sesiones. Cada nivel de análisis sirve al investigador de forma particular.

Los análisis continuos son la base de las decisiones que se toman en la planificación y desarrollo de cada sesión con los estudiantes, permiten probar las hipótesis y promover el desarrollo. Un aspecto clave es la generación y modificación, por parte del investigador, de los modelos relativos al conocimiento, acciones y disposiciones de los estudiantes. El análisis retrospectivo implica una reexaminación de un gran cuerpo de información. Este implica una revisión estructurada de las grabaciones del experimento de enseñanza, con el propósito de desarrollar modelos explicativos acerca del desarrollo del conocimiento matemático de los estudiantes.

Construcción del modelo: el fin del experimento de enseñanza es la elaboración de un modelo explicativo del desarrollo del conocimiento matemático de los estudiantes. Estos modelos explicativos empiezan a ser esbozados durante los análisis continuos, sin embargo, es durante el análisis retrospectivo cuando son articulados con más detalle. De acuerdo con Molina et al. (2011) el objetivo último del experimento de enseñanza es: "Elaborar un modelo del aprendizaje y/o desarrollo de los alumnos, en relación con un contenido específico, entendiendo este aprendizaje como resultado de la manera de operar y las situaciones puestas en juego por el investigador-docente" (p.79).

Generación y prueba de hipótesis: el experimento de enseñanza conlleva ciclos continuados de generación y prueba de hipótesis. Las conjeturas actuales de los investigadores guían sus interacciones con los estudiantes. Tales interacciones proporcionan información que respalda o genera modificaciones de esas suposiciones y también promueve la elaboración de nuevas conjeturas. Las hipótesis iniciales del equipo investigador guían el desarrollo del plan inicial de investigación. 
Grabación: la grabación de las sesiones con los estudiantes usualmente se complementa con la videograbación. La transcripción de las grabaciones en audio y video es esencial en el análisis posterior. Frecuentemente, las grabaciones se escuchan o se miran en medio de las sesiones, para apoyar los análisis continuados y la toma de decisiones.

\section{Contribuciones de los experimentos de enseñanza de "todo el grupo" a los experimentos TDE}

En los trabajos de Cobb (2000) y Cobb y Yackel (1996), se ha propuesto una metodología basada en los experimentos de enseñanza, para estudiar el aprendizaje de las matemáticas en las aulas. A continuación se presentan algunas características de la metodología de los experimentos de enseñanza de "todo el grupo", que se incorporan a los experimentos TDE.

Perspectiva emergente: esta perspectiva social y cognitiva proporciona los fundamentos teóricos de cada aspecto de la metodología TDE (Cobb, 2000). Desde tal enfoque el aprendizaje puede visualizarse como un proceso cognitivo del individuo y como un proceso social de un grupo. La relación básica entre las actividades constructivas de los estudiantes y el proceso social en el cual ellos participan, es reflexiva y no se atribuye algún tipo de preferencia entre ambos procesos. En esta perspectiva se considera que cuando los estudiantes reorganizan las actividades matemáticas individualmente, contribuyen al desarrollo de las prácticas matemáticas de la clase. A la inversa, las formas en las cuales hacen esas reorganizaciones, están determinadas por su participación en el desarrollo de las prácticas de clase (Cobb, 2000)

Así, esta perspectiva evita el debate sobre si el aprendizaje es principalmente individual o social. En su lugar, se afirma la utilidad de coordinar el análisis que resulta al considerar cada uno de estos aspectos.

Marco para el análisis: Cobb (2000) ha desarrollado un marco interpretativo para analizar la actividad individual y colectiva en el aula. El marco enfatiza la complementariedad de un foco sobre las normas y prácticas de la clase con un foco sobre los individuos, en relación con sus roles (profesor-estudiantes) y las concepciones que tienen sobre el contenido matemático. Tal investigación implica poner atención a la actividad individual, 
de pequeños equipos, de todo el grupo, sobre el contenido y sobre las conversaciones explícitas de los participantes en relación con el aprendizaje.

Enmarcar casos: Cobb (2000) sugiere una forma útil de pensar acerca de los productos de un experimento de "todo el grupo". Uno de los propósitos principales de este análisis es ubicar los eventos de la clase en un contexto teórico más amplio, enmarcándolos como casos particulares de un fenómeno más global. Esta perspectiva sugiere una posible vía para contrastar el análisis retrospectivo con el análisis "entre sesiones".

Según Simon (2000), la idea de "enmarcar casos" es un constructo frecuentemente usado en muchos tipos de investigaciones cualitativas. Se sugiere que los investigadores examinan la situación particular que han documentado, para identificar situaciones en las cuales desarrollar una explicación teórica que pueda orientar cuestiones relevantes en la investigación en educación matemática, y (o) en las comunidades de enseñanza.

\section{Fases de los experimentos de enseñanza}

Cobb y Gravemeijer (citados en Molina et al., 2011) distinguen 3 fases en el desarrollo de los experimentos de enseñanza: preparación del experimento, experimentación y análisis retrospectivo de los datos. En la Tabla 1 se presentan las acciones implicadas en cada una de las fases de los experimentos de enseñanza recogidas por Molina et al. (2011). 
Tabla 1

Acciones contempladas en cada fase de los experimentos de enseñanza

Fase I. Preparación de la experimentación

- Definir el problema de investigación.

- Justificar el interés y la necesidad de realizar este estudio.

- Elegir, justificar la elección y describir a los sujetos participantes en el estudio.

- Diseñar, en líneas generales, en qué va a consistir la secuencia de intervenciones en el aula y justificar dicho diseño.

- Identificar metodologías de enseñanza adecuadas para el contenido a abordar en el aula, según los objetivos de investigación planteados.

- Elaborar hipótesis de investigación relativas al problema en estudio, que puedan ser contrastadas a partir de las intervenciones en el aula.

- Identificar los objetivos concretos de la intervención por realizar.

- Diseñar la siguiente intervención teniendo en cuenta: el análisis de los datos ya recogidos, la búsqueda bibliográfica realizada, los conocimientos previos de los alumnos y el trabajo efectuado en el aula fuera de las sesiones de recogida de datos del estudio.

- Justificar el diseño y la temporalización de la intervención, en especial las decisiones tomadas a partir del análisis de los datos ya recogidos.

- Elaborar hipótesis sobre los resultados por obtener en la intervención por realizar. Intentar prever las posibles reacciones de los alumnos y las dificultades que puedan presentarse.

II Fase. Experimentación y análisis preliminar

- Realizar una recogida de datos exhaustiva mediante grabaciones en video o en audio, recogida de las hojas de trabajo de los alumnos, toma de notas por un observador, etc.

- Modificar de forma justificada el diseño de la intervención, si se considera conveniente de acuerdo con los objetivos concretos de la intervención.

- El investigador-docente, si lo considera necesario, tomará notas sobre la intervención que complementen y ayuden a comprender los datos recogidos.

- Analizar los datos recogidos en el aula.

- Contrastar los resultados con las hipótesis previamente elaboradas sobre esa intervención y, en su caso, reformular alguna de las hipótesis por tener en cuenta en futuras intervenciones.

III Fase. Análisis retrospectivo de las sesiones

- Realizar la trascripción de las grabaciones realizadas.

- Organizar los datos recogidos.

- Analizar todos los datos recogidos de forma conjunta (utilizar la información obtenida en la búsqueda bibliográfica previamente realizada para guiar dicho análisis).

- Dar respuesta, si es posible, a los objetivos del estudio.

- Contrastar los resultados con los obtenidos en otros estudios.

- Elaborar un modelo que describa el aprendizaje o desarrollo de los alumnos, de los docentes o de las tareas realizadas, de los cambios que son considerados aprendizaje o desarrollo de los alumnos a lo largo del experimento de enseñanza, entendiendo estos como ocasionados por las maneras de operar y las situaciones puestas en juego por el investigador-docente.

- Si es necesario, se ampliará la búsqueda bibliográfica realizada.

Fuente: tabla adaptada de Molina et al. (2011) 


\section{Análisis de la información recogida en los experimentos de enseñanza TDE}

Como consecuencia del carácter cíclico de los estudios de diseño, se hacen necesarios 2 tipos de análisis de datos: uno continuo, que se efectúa después de cada sesión, y uno final de todos los datos recogidos en el proceso de investigación. Las cuestiones a las que da respuesta el "análisis entre sesiones" son típicamente de carácter práctico y están relacionadas con el objetivo de promover el aprendizaje de los estudiantes participantes. Al finalizar la experimentación se lleva a cabo el análisis retrospectivo de la intervención de enseñanza (Molina et al., 2011). Simon (2000) sostiene que las cuestiones que emergen como significativas en los análisis continuos que se realizan después de cada sesión, llegan a constituirse en focos del estudio final de los datos.

La metodología TDE se inclina por proporcionar una perspectiva dual sobre el desarrollo del conocimiento del profesor, coordinando análisis sobre el conocimiento individual y grupal. Delimita inicialmente este marco en términos muy generales, sugiriendo que el análisis del desarrollo del conocimiento grupal se realiza con base en la información recogida durante la experiencia colectiva, que supone estudiar las concepciones de los estudiantes en relación con el contenido matemático, así como también el desarrollo de las prácticas sociales. El análisis del conocimiento individual efectúa mediante el estudio de casos, el cual ha de considerar el contexto social dentro del cual ha tenido lugar el desarrollo del conocimiento individual.

Las aportaciones iniciales de Simon, acerca de la elaboración de un marco de referencia para el análisis de la información del experimento de enseñanza, han sufrido modificaciones que han incidido en el progreso de su propuesta. En los orígenes del experimento de enseñanza sobre el desarrollo del conocimiento, el profesor Simon consideró la perspectiva emergente (Cobb, 2000) como marco de referencia para el análisis de la información.

La perspectiva emergente se basa en la coordinación de las perspectivas social y cognitiva y fue desarrollada para caracterizar el aprendizaje de las matemáticas en el aula (Cobb y Yackel, 1996). En el caso de las teorías social y cognitiva, los investigadores han señalado su complementariedad y arguyen que cada una tiene sus fortalezas y limitaciones que las hace más útiles en algunos trabajos y menos en otros (Simon, 2012). Una perspectiva social se utiliza para caracterizar el aprendizaje cuando la unidad de 
análisis es la clase (incluyendo al profesor). Una perspectiva cognitiva se emplea cuando la unidad de análisis son estudiantes individuales. El uso de diferentes herramientas teóricas para analizar esas distintas unidades de análisis proporciona a la teoría una cierta elegancia y claridad (Simon, 2012). El análisis de observaciones de clases se concentra en la búsqueda de normas emergentes -sociales y sociomatemáticas- y la identificación de una secuencia de prácticas matemáticas desarrolladas a lo largo del tiempo. Esos análisis son coordinados con análisis de datos procedentes de entrevistas a estudiantes individuales. Los datos de la entrevista son analizados usando una perspectiva constructivista, identificando las concepciones de los estudiantes en relación con diferentes aspectos del experimento de diseño realizado (Cobb, 2003).

Sin embargo, con el propósito de obtener descripciones más finas sobre los conocimientos matemáticos puestos de manifiesto por los futuros profesores, Simon (2012) y sus colaboradores han trazado una nueva manera de visualizar el análisis de la información procedente de los experimentos de enseñanza TDE.

Movidos por el interés en comprender el proceso que tiene lugar cuando los futuros profesores de matemáticas van de una práctica matemática a otra, se han planteado ¿qué herramientas teóricas pueden ser utilizadas para este propósito? Considerando esto, han empezado por tomar en cuenta teorías que permitan abordar la siguiente distinción: lo que se usa para mirar (el lente teórico) y lo que se mira (un individuo o un grupo). Tal distinción les ha permitido ir más allá en el uso tradicional de teorías sociales para estudiar datos de colectivos (grupos) y teorías cognitivas para estudiar datos individuales. La propuesta de análisis de estos investigadores se describe en la Tabla 2.

\begin{tabular}{|cc|}
\hline \multicolumn{2}{|c|}{ Tabla 2 } \\
Propuesta de análisis, según sujeto y naturaleza \\
\hline Análisis cognitivo & Análisis social \\
\hline Análisis cognitivo individual & Análisis social del individuo \\
Análisis cognitivo del grupo & Análisis social del grupo \\
\hline
\end{tabular}

Fuente: tabla adaptada de Simon (2012)

De acuerdo con Simon (2012), el análisis cognitivo de un individuo (cuadrante superior izquierdo) y el análisis social del grupo (cuadrante inferior derecho) requieren poca elaboración, ya que es común para los investigadores realizar análisis cognitivos del pensamiento matemático de los individuos (ej. constructivismo), y análisis sociales de la 
comunicación matemática dada en la discusión en pequeños grupos o en todo el grupo (ej. teoría sociocultural, interaccionismo simbólico). Sin embargo, los otros 2: análisis social de un individuo (cuadrante superior derecho) y análisis cognitivo del grupo (cuadrante inferior izquierdo), ameritan cierta discusión.

Análisis social de un individuo: en los análisis caracterizados por este cuadrante, el investigador considera que la actividad de un estudiante trabajando solo es influenciada por las normas y prácticas de su clase de matemáticas, el lenguaje que utiliza, las prácticas culturales de su familia, etc. Tales explicaciones sociales pueden ser útiles para comprender aspectos de la actividad y aprendizaje del individuo.

Análisis cognitivo de un grupo: el argumento para el análisis cognitivo de la actividad grupal es paralelo al planteado para el análisis social de la acción individual; el análisis cognitivo usa conocimientos y constructos adecuados para ampliar lo que se informa y para generar explicaciones útiles relacionadas con los datos. Mientras que desde una perspectiva social, una conversación podría ser vista como una negociación de significados o como un aumento de la participación en las prácticas del grupo, esta ha resultado ventajosa para ver cómo 2 o más estudiantes con diferentes concepciones intentan comprender las ideas de otros.

Según Martin Simon, en el contexto de las teorías orientadas cognitivamente, hay una extensa base de conocimiento sobre distinciones de las concepciones de los estudiantes; él y sus colaboradores han empleado análisis cognitivos para comprender el proceso de aprendizaje del grupo, haciendo uso de resultados empíricos obtenidos en trabajos previos, en relación con las concepciones de los individuos sobre contenidos y procesos matemáticos (Simon y Blume, 1996; Simon, Tzur, Heinz, Kinzel y Smith, 2000).

\section{Reflexiones finales}

Este artículo se ha centrado en mostrar los fundamentos teóricos que subyacen a la investigación de diseño y los experimentos de enseñanza. Estos principios se han aplicado en el desarrollo de una investigación realizada por la autora de este artículo, en la que se abordó el problema de promover el desarrollo del conocimiento matemático de futuros docentes de educación primaria, respecto a las nociones razón y proporcionalidad (Valverde, 2012); no ha sido el objetivo de este artículo describir tal estudio. Sin embargo, la experiencia con la metodología TDE permite a la autora reconocer su potencial y limitaciones en el marco de la investigación referenciada. 
Como fortaleza, se reconoce que los experimentos de enseñanza se constituyen en una poderosa opción para estudiar el desarrollo del conocimiento del futuro docente. En la investigación desarrollada por la autora se recogió una extensa cantidad de información contextualizada acerca de la comprensión y negociación de ideas matemáticas asociadas a la razón y la proporcionalidad, a partir de la cual se elaboraron algunos aportes teóricos acerca de este dominio de aprendizaje. Como afirman Cobb et al. (2003), la riqueza de tales estudios radica en que al basarse en hechos empíricos, son esenciales para la mejora de la educación.

Estas investigaciones se realizan desde un enfoque metodológico que procura relacionar la teoría y la práctica. En el estudio efectuado por la autora se ha experimentado un continuo ir y venir entre ambas. Se recurrió a la teoría para interpretar, entre otros, el desempeño de los estudiantes, y enmarcarlo dentro de comprensiones amplias relacionadas con fenómenos del aprendizaje de la razón y la proporcionalidad. Por otro lado, se logró describir y explicar, presumiblemente, desempeños que no están recogidos en otras investigaciones. Específicamente, se describen 107 indicadores de actuación relacionados con el conocimiento matemático sobre la proporcionalidad, detectados en el análisis de las transcripciones orales del trabajo colaborativo en 6 de las tareas. Destaca, como aporte de la investigación, la descripción de acciones vinculadas al razonamiento proporcional y a la comprensión de la proporcionalidad en el contexto de la formación de maestros, pues en la bibliografía precedente se encuentra la descripción de una gran variedad de manifestaciones de niños o estudiantes de secundaria, y muy poca de maestros en formación inicial.

Otra de las bondades que la autora reconoce en la metodología TDE, es que la claridad de las acciones por realizar en cada fase del experimento, contribuye en gran medida en la concepción, aplicación y análisis de la experiencia de aula. Tales acciones no surgen por voluntad de la investigadora, sino que responden a una organización de lineamientos bien definidos en el diseño de este tipo de estudios.

La metodología de investigación TDE conlleva, además del análisis previo y retrospectivo, el análisis del trabajo desarrollado socialmente y del expuesto individualmente. Esta doble perspectiva posibilita generar una panorámica más completa de lo sucedido en la experimentación, y de cómo funciona el diseño en ambas facetas.

Es preciso destacar que los análisis previos realizados después de las sesiones, se centran en la toma de decisiones en beneficio de la mejora del diseño y del aprendizaje 
de los estudiantes. También es necesario indicar que en este tipo de estudios se hace imprescindible contar con diversas técnicas e instrumentos de recogida de los datos. Las producciones escritas han de respaldarse con grabaciones en video o audio, decisión que incide en la riqueza de lo manifestado por los participantes. Es claro que recoger información a través de distintos registros: producciones escritas, grabaciones de audio y videograbación, afecta la validez de los resultados reportados, pues el registro en papel se usa para constatar las actuaciones identificadas en las transcripciones del trabajo colaborativo y en sentido inverso. Sin embargo, es preciso reconocer la dificultad que entraña la transcripción e interpretación de las producciones orales.

Otra recomendación que se desprende de la experiencia de la autora como investigadora principal en un experimento TDE, se refiere a la constante permanencia en el contexto investigado. En el estudio realizado ha sido oportuno el hecho de que la investigadora haya permanecido en el aula desde el primer día de clase y compartido con los estudiantes el trabajo de la asignatura en todas las sesiones. Esta permanencia permite conocer la dinámica de trabajo seguida regularmente y también con detalle, los contenidos y actividades tratados entre las sesiones previas y posteriores, lo cual permite generar explicaciones sobre posibles influencias en el desempeño manifestado por los estudiantes.

\section{Referencias}

Brown, Ann. (1992). Design Experiments: Theoretical and methodological challenges in creating complex interventions in classroom settings. Journal of the Learning Sciences, 2(2), 141-178.

Cobb, Paul. (2000). Conducting teaching experiments in collaboration with teachers. En Anthony Kelly y Richard Lesh (Eds.), Handbook of research design in mathematics and science education (pp. 307-333). Mahwah, NJ: Lawrence Erlbaum Associates.

Cobb, Paul. (2003). Investigating students' reasoning about linear measurement as a paradigm case of design research. En Michelle Stephan, Janet Bowers y Paul Cobb (Eds.), Supporting students' development of measuring conceptions: Analyzing students' learning in social context (Journal for Research in Mathematics Education Monograph; No 12, pp. 1-16). Reston, VA: NCTM.

Cobb, Paul y Steffe, Leslie. (1983). The constructivist researcher as teacher and model builder. Journal for Research in Mathematics Education, 14(2), 83-94.

Cobb, Paul y Yackel, Erna. (1996). Constructivist, emergent, and sociocultural perspectives in the context of developmental research. Educational Psychologist, 31(3/4), 175-190. 
Cobb, Paul, Confrey, Jere, DiSessa, Andrea, Lehrer, Richard y Schauble, Leona. (2003). Design experiment in Educational Research. Educational Researcher, 32(1), 9-13.

Cohen, Louis y Manion, Lawrence. (2002). Métodos de investigación educativa. Madrid: La Muralla.

Collins, Allan, Joseph, Diana y Bielaczyc, Katherine. (2004). Design research: Theoretical and methodological issues. Journal of the Learning Sciences, 13(1), 15-42.

Confrey, Jere. (2006). The evolution of design studies as methodology. En R. Keith Sawyer (Ed.), The Cambridge Handbook of the Learning Sciences (pp. 135-152). New York, NY: Cambridge University Press.

Confrey, Jere y Lachance, Andrea. (2000). Transformative teaching experiments through conjecture-driven research design. En Anthony Kelly y Richard Lesh (Eds.), Handbook of research design in mathematics and science education (pp. 231-266). Mahwah, NJ: Erlbaum.

Design-Based Research Collective (2003). Design-Based Research: An Emerging Paradigm for Educational Inquiry. Educational Researcher, 32(1), 5-8.

Gravemeijer, Koeno. (1994). Educational development and developmental research. Journal for Research in Mathematics Education, 25(5), 443-471.

Kelly, Anthony y Lesh, Richard. (2000). Handbook of research design in mathematics and science education. New Jersey, NJ: Lawrence Erlbaum Associates.

Molina, Marta. (2007). Desarrollo del pensamiento relacional y comprensión del signo igual. (Tesis para optar por el grado de doctora en Didáctica de la Matemática). Departamento de Didáctica de la Matemática. Universidad de Granada, España.

Molina, Marta, Castro, Encarnación, Molina, José y Castro, Enrique. (2011). Un acercamiento a la investigación de diseño a través de los experimentos de enseñanza. Enseñanza de las Ciencias, 29(1), 75-88.

Simon, Martin y Blume, Glendon. (1996). Justification in the mathematics classroom: A study of prospective elementary teachers. Journal of Mathematical Behavior, 15(1), 3-31.

Simon, Martin. (2000). Research on the development of mathematics teacher: The teacher development experiment. En Anthony Kelly y Richard Lesh (Eds.), Handbook of research design in mathematics and science education (pp. 335-359). Mahwah, NJ: Lawrence Erlbaum Associates.

Simon, Martin. (2012). Extending the coordination of cognitive and social perspectives. PNA, 6(2), 43-49.

Simon, Martin, Tzur, Ron, Heinz, Karen, Kinzel, Margareth y Smith, Margareth. (2000). Characterizing a perspective underlying the practice of mathematics teachers in transition. Journal for Research in Mathematics Education, 31(5), 579-601. 
Steffe, Leslie y Thompson, Patrick (2000). Teaching experiment methodology: Underlying principles and essential elements. En Anthony Kelly y Richard Lesh (Eds.), Handbook of research design in mathematics and science education (pp. 267-306). Mahwah: NJ: Lawrence Erlbaum Associates.

Tabak, Iris. (2004). Reconstructing context: Negotiating the tension between exogenous and endogenous educational design. Educational Psychologist, 39(4), 225-233.

Valverde, Gabriela. (2012). Competencias matemáticas promovidas desde la razón y la proporcionalidad en la formación inicial de maestros. (Tesis para optar por el grado de doctora en Didáctica de la Matemática). Universidad de Granada, España. Recuperada de http://hera.ugr.es/tesisugr/21402334.pdf 